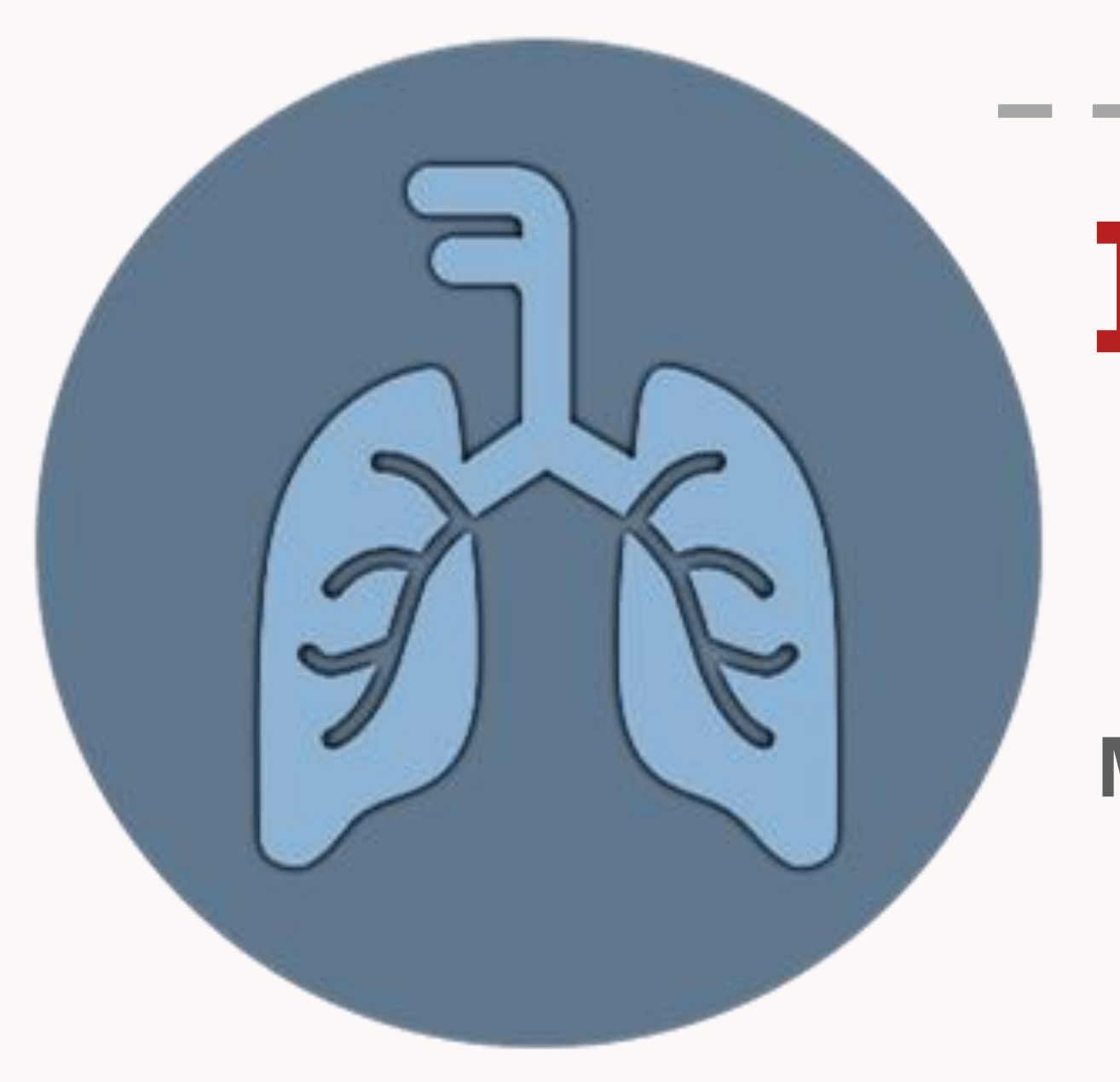

\title{
Influenza, RSV, and Other Respiratory Infections among Children in Qatar
}

Maria K. Smatti ${ }^{1}$, Hamad Al Romaihi ${ }^{2}$, Hebah Al-Khatib ${ }^{3}$, Peter Coyle ${ }^{4}$, Asmaa Al Thani ${ }^{1}$, Muna Al Maslamani ${ }^{4}$, Hadi M. Yassine ${ }^{1^{*}}$ 'Biomedical Research Center, ${ }^{2}$ Ministry of Public Health, ${ }^{3}$ Hamad Bin Khalifa University, ${ }^{4}$ Hamad Medical Corporation

\section{Background}

Acute respiratory infections (ARIs) lead to high rates of mortality and morbidity among children. According to the $\mathrm{WHO}$, respiratory infections were responsible for about a million deaths in children younger than four years old during 2017. The most commonly reported virus is respiratory syncytial virus (RSV). Other common viruses include Influenza A and B viruses, parainfluenza viruses (PIVs), adenovirus, and human rhinovirus (HRV), human metapneumovirus (HMPV), human coronavirus (HCoV) and human bocavirus (HboV). Importantly, studies on the etiology of respiratory infections among children in Qatar and surrounding countries are still limited.

\section{Research goal}

To describe the prevalence and seasonal variation of RSV, influenza, and other respiratory infections circulating among children with influenza like illness (ILI) in Qatar.

\section{Methodology}

\section{ำ}

33,404 ILI patients

( $\leq 15$ years) , 2012-2017

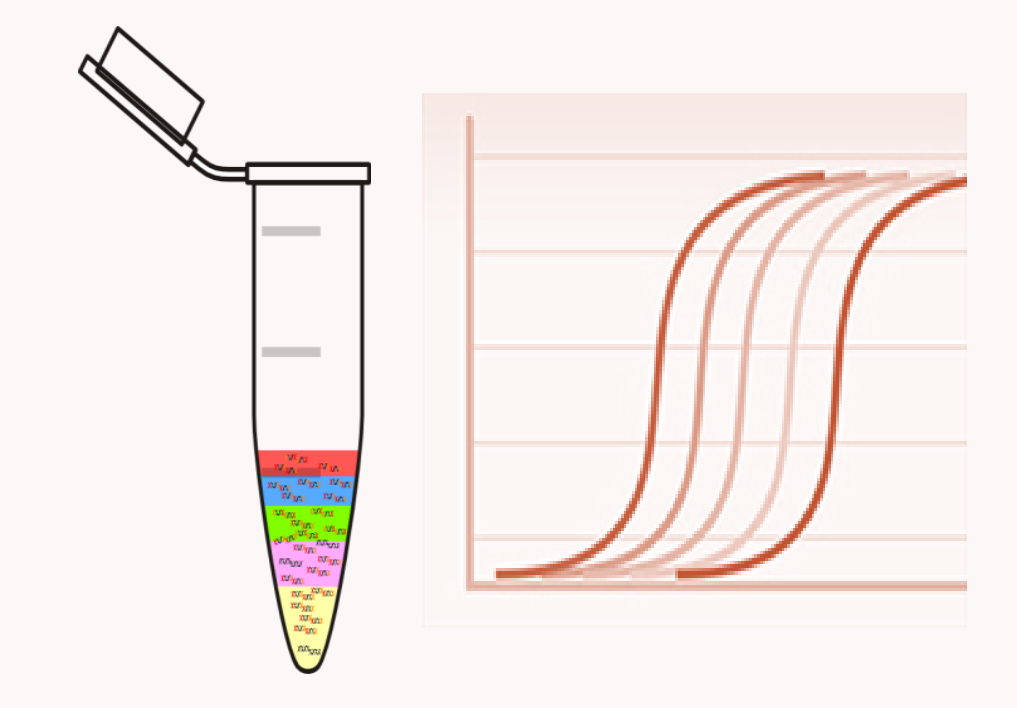

Molecular Detection

(panel of 21 viruses)

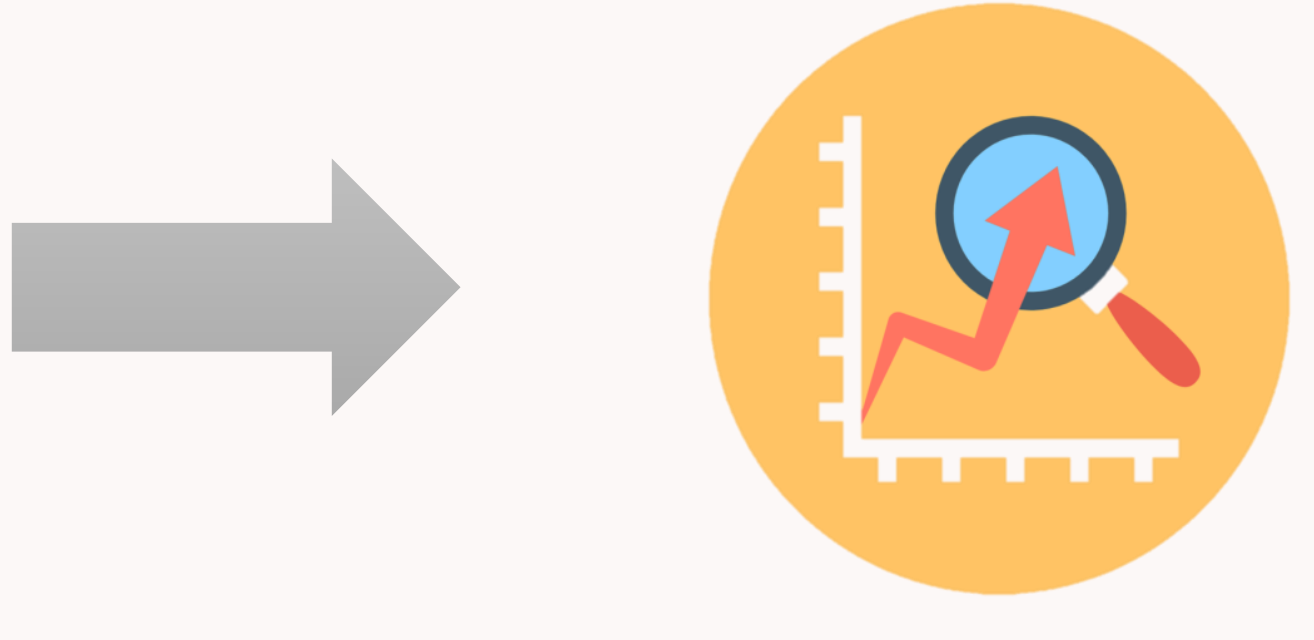

Statistical Analysis

Significant at $p$-value $<0.05$

\section{Findings}

1- Overall prevalence

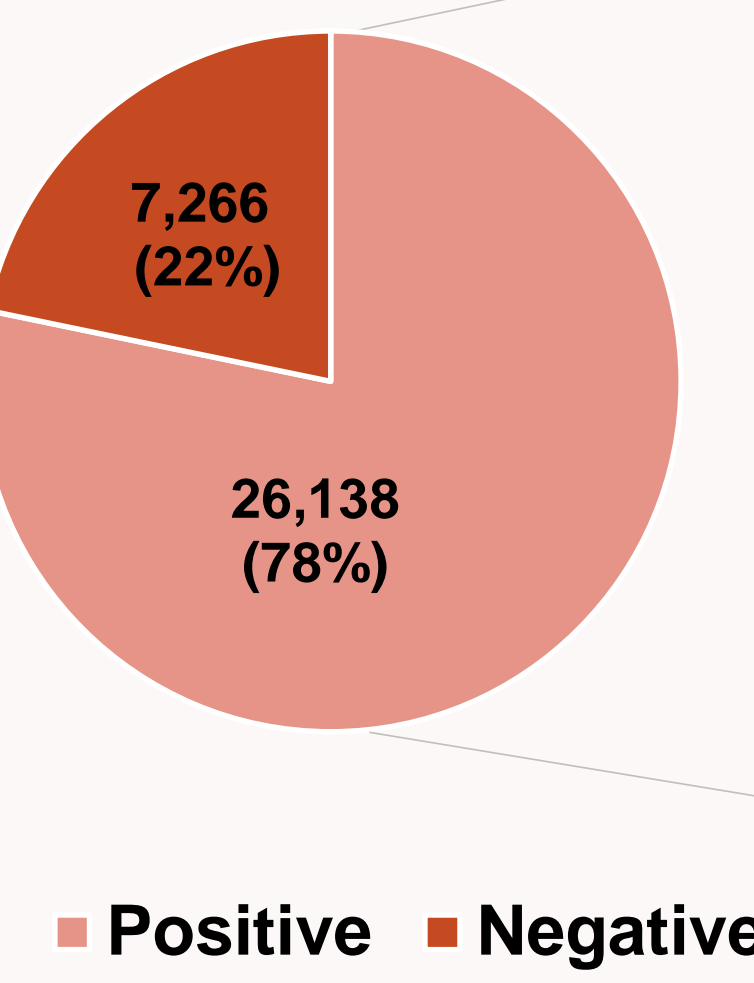

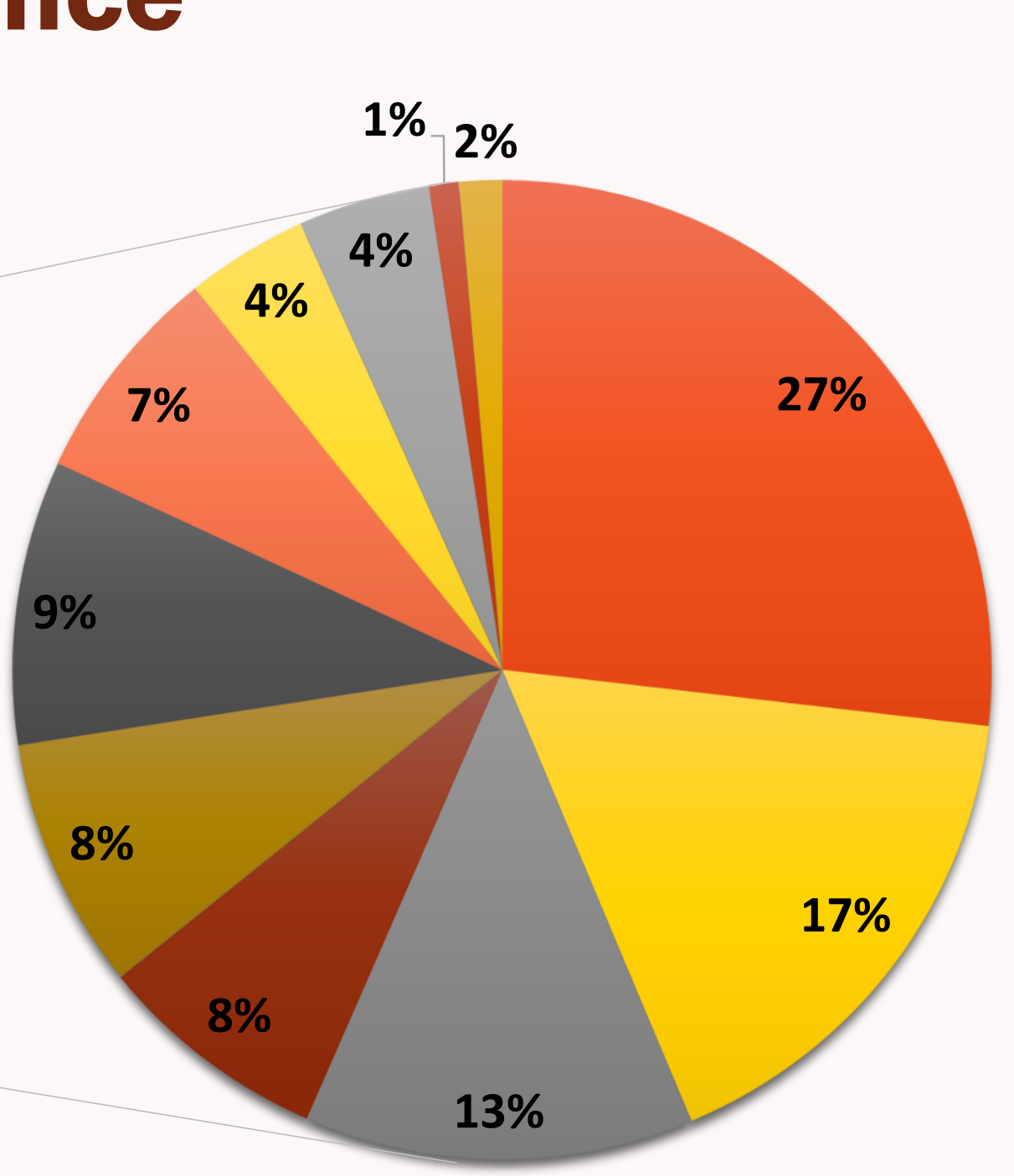

HRV

RSV

Influenza

- Adenovirus

HBoV

- Coronavirus

PIVs

HMPV

- Enterovirus

- Parechovirus

M. pneumoniae

\section{2- Annual rate of infection}

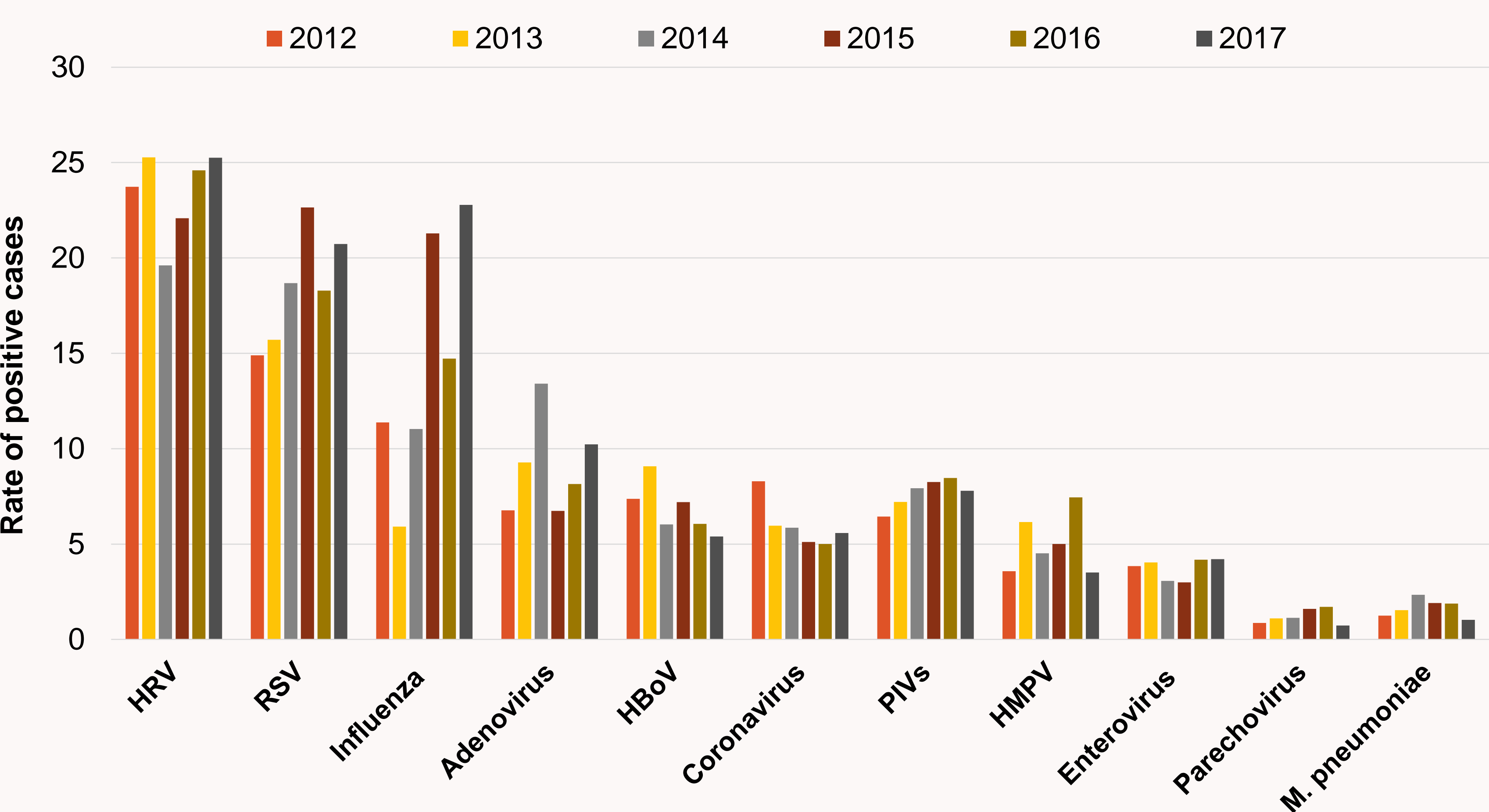

3- Infection rates among different age groups

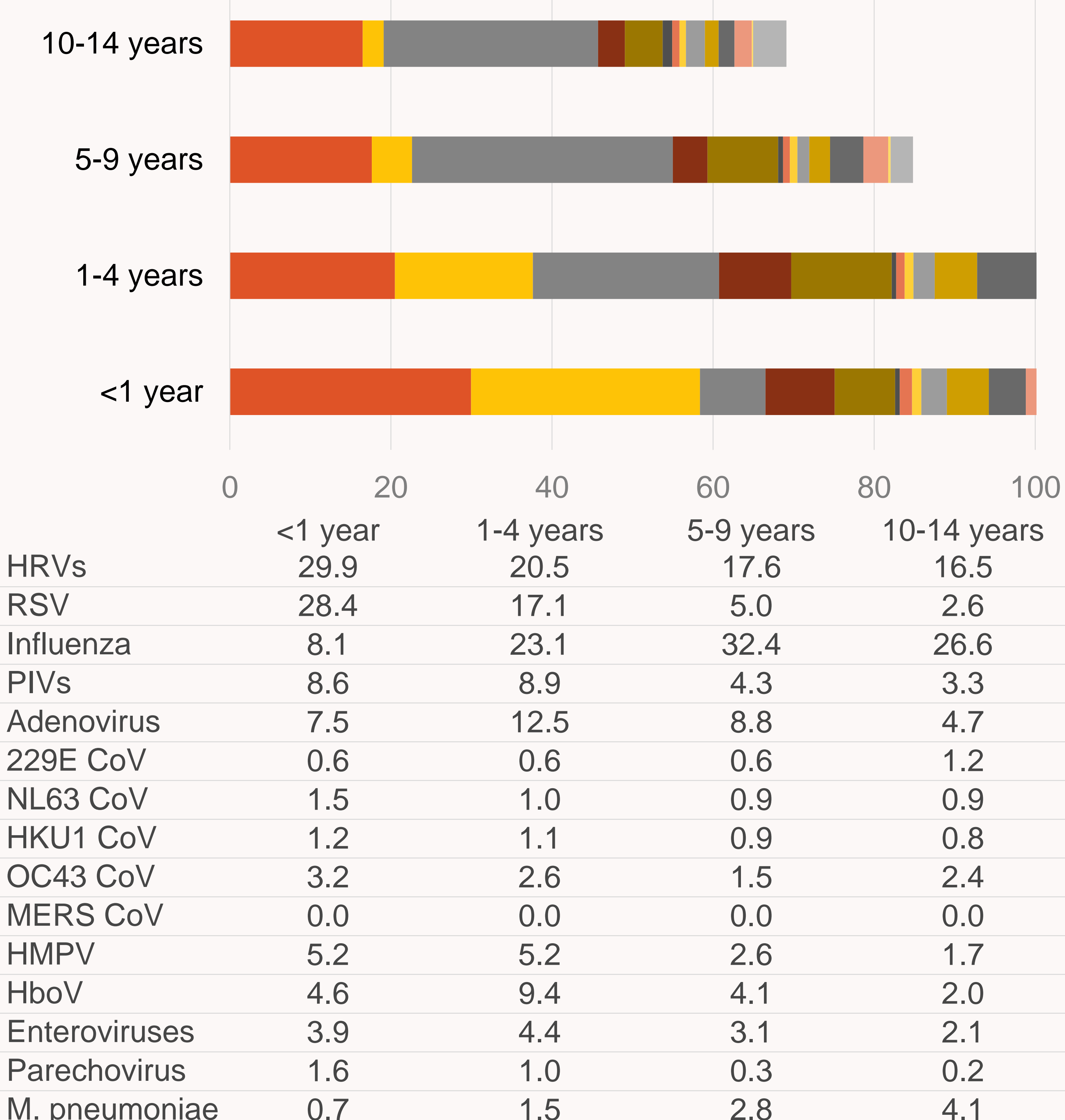

\section{3- Seasonality}

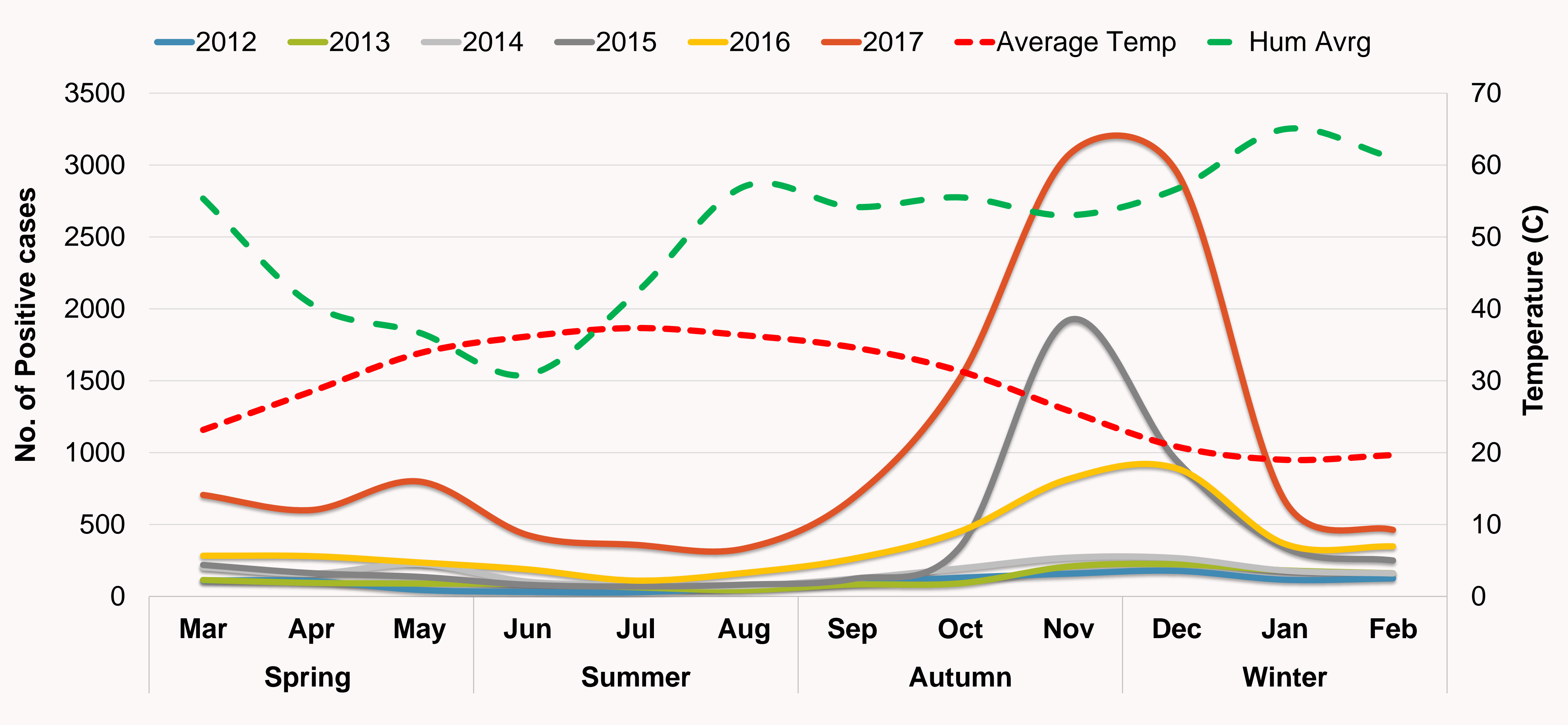

4- Infection rates among males and females

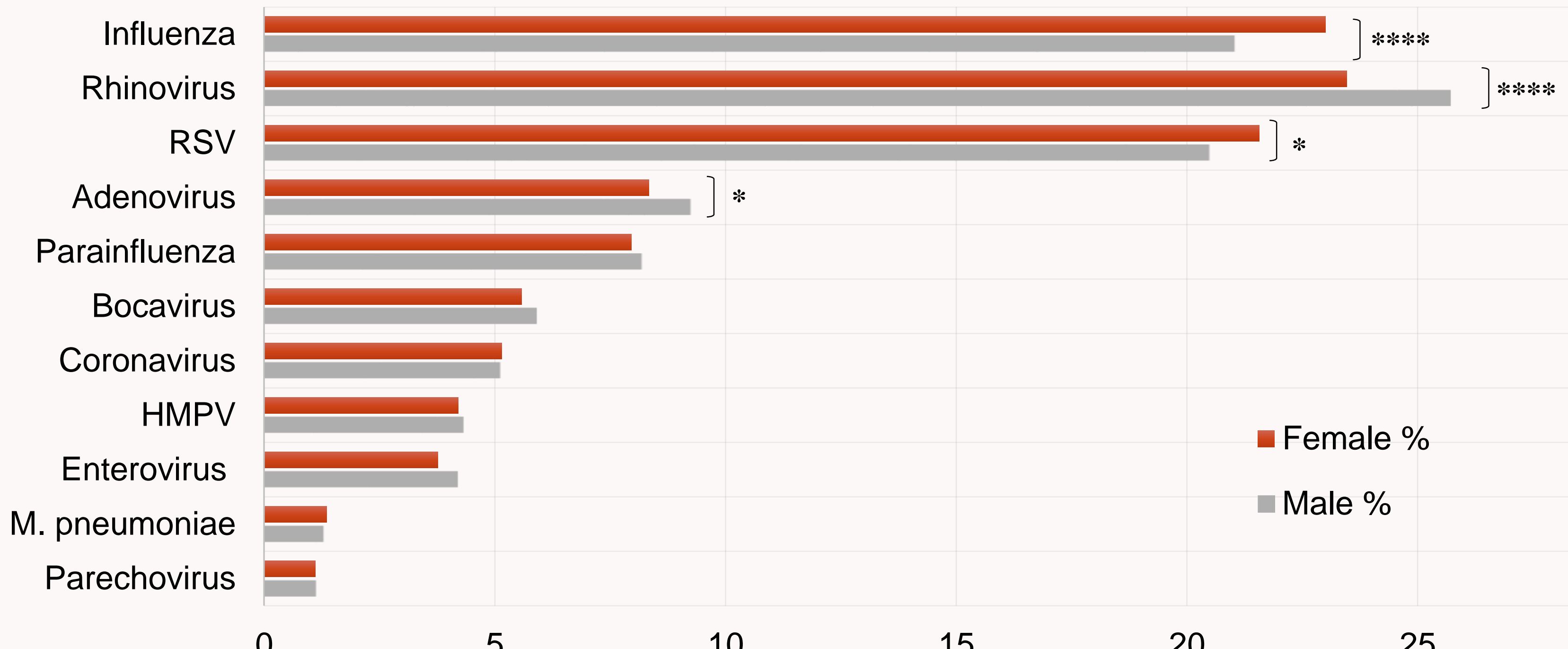

\section{(1) Conclusion}

$>$ At least one respiratory pathogen was detected in $78 \%$ of ILI patients.

$>\mathrm{HRV}, \mathrm{RSV}$, and influenza viruses are responsible for two-thirds of cases.

$>$ RSV, influenza, and HMPV circulated in winter, while HRV was highly active during other seasons.

$>$ Gender-dependent differences affect infection rates.

$>$ This is the largest scale report on ILIs among children in the MENA region, and thus, provides better insights on respiratory pathogens circulation. 